\title{
Sistemas de navegação indoor e sistema de compras para pessoas com deficiência visual: potenciais no uso em supermercado
}

Indoor navigation system and shopping for blind people: potentials in supermarket use

Tainá A. Bueno de Oliveira ${ }^{1}$

Flávio Anthero N. V. dos Santos ${ }^{2}$

Milton José Cinelli ${ }^{3}$ 


\section{Resumo}

Pessoas com deficiência visual na realização de tarefas como se locomover e pegar objetos, o cego utiliza uma visualização mental da rota de forma já memorizada, contudo, para isso, é necessário que haja uma instrução prévia, um conhecimento do local. Nesse contexto, buscouse explorar e discutir TA's que estão sendo testadas de localização indoor e identificação de produtos, com o intuito de descobrir potenciais no uso em supermercados. Utilizou-se RBS de forma exploratória, a fim de definir as tecnologias que seriam pesquisadas e o recorte foi na análise comparativa de Beacons, RFID e QR Code, citadas pela literatura. Como resultado, todos os artigos apresentaram potencialidades no uso dessas tecnologias em ambiente de supermercado e na identificação de produtos da gôndola.

Palavras-chave: Navegação Indoor, Tecnologia Assistiva, Compras em Supermercado.

\section{Abstract}

People with visual impairments in performing tasks such as getting around and picking up objects, the blind uses a mental visualization of the route in a way already memorized, however, for this, it is necessary that there is a prior instruction, a knowledge of the place. In this context, we sought to explore and discuss AT's that are being tested for indoor location and identification of products, in order to discover potentials in use in supermarkets. A systematic literature review was used in an exploratory way in order to define the technologies that would be researched and the cut was in the comparative analysis of Beacons, RFID and QR Code, cited in the literature. As a result, all the articles presented potential in the use of these technologies in a supermarket environment and in the identification of products from the shelf.

Key-words: Indoor navigation, Assistive Technology, Supermarket Shopping.

\footnotetext{
1 Mestranda em Design no PPGDesign/UDESC, SC,

Brasil,tainabueno@gmail.com

2 Dr. , UDESC, SC, Brasil,flavioanvs@hotmail.com

3 Dr. , UDESC, SC, Brasil, milton.cinelli@udesc.br
} 


\section{Introdução}

É importante para o ser humano mover-se ao longo de um ambiente de forma livre e sem preocupação, independente de sua limitação física ou da dificuldade que o ambiente apresenta. A navegação envolve identificar uma boa rota, localizar e evitar obstáculos, localizar entradas e saídas como portas e banheiros e também a identificação do local atual.

Para pessoas com deficiência visual, normalmente o que se usa é uma visualização mental da rota de forma já memorizada, porém para isso é necessário que haja uma instrução prévia, um conhecimento do local. Contudo, quando esse conhecimento prévio não é possível, andar por ambientes é uma tarefa desafiadora, principalmente quando estes são ambientes de grande circulação, como ambientes públicos, hospitais, supermercados, pontos turísticos, muitas vezes sendo um desafio até mesmo para pessoas videntes. Para transitar em ambientes como estes, é necessário o uso de pistas direcionais ou mapas para chega-se ao destino. O GPS (Global Positioning System), comumente usado em dispositivos mobile, embora possa localizar e informar as pessoas em locais externos, não faz os direcionamentos de ambientes internos. Por outro lado, existe uma tecnologia conhecida como IPS (Indoor Positioning System), que funciona por ondas de rádio entre outras informações sensoriais e é utilizado para localização e identificação de objetos em ambientes internos.

$\mathrm{Na}$ busca da promoção da autonomia de pessoas com deficiência visual em compras de supermercado, procura-se investigar tecnologias que possam agir como facilitadoras neste processo. Sistemas que trabalham em conjunto com dispositivos móveis, emitindo mensagens para celulares, por exemplo, podem auxiliar nessa dificuldade relatada. Atualmente, os celulares com sistemas operacionais mais comuns, como Android e iOS, possuem leitores de tela bastante eficientes, podendo, assim, fazer a leitura das mensagens e repassá-las no formato de áudio. Contudo, esses sistemas são pouco conhecidos e utilizados no Brasil, principalmente quando se trata da utilização na área comercial em forma de Tecnologia Assistiva com o intuito de facilitar o acesso à locais e à produtos. Nessa área de pesquisa, alguns experimentos já foram publicados, porém, até o presente não foi documentado nenhum estudo no Brasil.

O presente artigo apresenta e discute algumas tecnologias que estão sendo testadas com o propósito de localização indoor e identificação de produtos. O principal objetivo do artigo é verificar quais dessas tecnologias apresentadas podem vir a ser utilizadas em supermercados no sentido de promover a inclusão e autonomia de pessoas com deficiência visual na localização precisa dentro do ambiente, além do reconhecimento de cada produto, para posteriormente poder adquiri-lo.

\section{REVISÃO BIBLIOGRÁFICA}




\subsection{Deficiência Visual}

Segundo estudo divulgado pela Organização Mundial de Saúde (OMS), estima-se que mais de um bilhão de pessoas em todo o mundo apresenta algum tipo de deficiência, e que, como revela o site Portal Brasil, uma em cada cinco tem sua vida dificultada por falta de condições. No Brasil, o Instituto Brasileiro de Geografia e Estatística (IBGE), no Censo de 2010, revelou que $23,9 \%$ da população, cerca de 45,6 milhões de pessoas, declararam ter pelo menos uma das deficiências investigadas (auditiva, visual, motora e mental ou intelectual). De acordo com o IBGE, deficiência visual foi a que mais se destacou sobre a população, com 35,7 milhões, atingindo $18,8 \%$. O Decreto 5.296 aprovado em 2 de dezembro de 2004 regulamenta sobre pessoas com deficiência. Nele é definida a deficiência visual como "Deficiência visual: cegueira, na qual a acuidade visual é igual ou menor que 0,05 no melhor olho, com a melhor correção óptica; a baixa visão, que significa acuidade visual entre 0,3 e 0,05 no melhor olho, com a melhor correção óptica; os casos nos quais a somatória da medida do campo visual em ambos os olhos for igual ou menor que 600; ou a ocorrência simultânea de quaisquer das condições anteriores". (BRASIL, 2004, art. 5).

As deficiências podem ser parte ou uma expressão de uma condição de saúde, mas não indicam, necessariamente, a presença de uma doença ou que o indivíduo deva ser considerado doente, conforme a Classificação Internacional de Funcionalidade, Incapacidade e Saúde (CIF, 2004, p.15). O que limita a vida de uma pessoa com deficiência é o meio no qual ela está inseri$\mathrm{da}$, e cabe às políticas públicas e ao coletivo fazer com que as oportunidades se equiparem às demais pessoas na sociedade, garantindo, assim, o acesso a bens e serviços. No entanto, para se garantir acessibilidade é preciso mudar a forma como se é pensada a necessidade de pessoas com deficiência. É preciso entender que a inclusão é sim um desejo, mas é também uma necessidade.

\subsection{Acessibilidade}

Acessibilidade é a palavra utilizada hoje para tratar, de forma simplificada, o acesso a variados lugares e a interação com objetos de pessoas com deficiência. As palavras acessibilidade e acessível pela definição do dicionário Michaelis Online: Acessibilidade, substantivo feminino: Facilidade de acesso, de obtenção; Facilidade no trato. Acessível, adjetivo masculino e feminino: De fácil acesso; A que se pode chegar; aproximável; que se pode alcançar, conseguir ou possuir; compreensível, inteligível.

Há também uma definição de acessibilidade, ajuda técnica e desenho universal voltada para as questões legislativas no Decreto 5.296/04, em seu artigo $8^{\circ}$ :

I - Acessibilidade: condição para utilização, com segurança e autonomia, total ou assistida, dos espaços, mobiliários e equipamentos urbanos, das edificações, dos serviços de transporte e dos dispositivos, sistemas e meios de comunicação e informação, por pessoa portadora de deficiência ou com 
mobilidade reduzida; [...].

V - Ajuda técnica: os produtos, instrumentos, equipamentos ou tecnologia adaptados ou especialmente projetados para melhorar a funcionalidade da pessoa portadora de deficiência ou com mobilidade reduzida, favorecendo a autonomia pessoal, total ou assistida; [...].

IX - Desenho universal: concepção de espaços, artefatos e produtos que visam atender simultaneamente todas as pessoas, com diferentes características antropométricas e sensoriais, de forma autônoma, segura e confortável, constituindo-se nos elementos ou soluções que compõem a acessibilidade. (BRASIL, 2004, art. 8ㅇ).

\subsection{Tecnologia Assistiva}

Segundo Bersch e Pelosi (2006), a Tecnologia Assistiva (TA) tem como principal objetivo proporcionar habilidades funcionais promovendo ou ampliando a melhora na inclusão social. "A Tecnologia Assistiva (TA) é uma área de conhecimento que engloba recursos e serviços com o objetivo de proporcionar ou ampliar habilidades funcionais de uma pessoa com deficiência ou com incapacidades advindas do envelhecimento. O objetivo da TA é o de promover a vida e a inclusão social de seus usuários". (BERSCH, PELOSI, 2006, p. 9)

A norma Internacional - International Organization for Standardization - ISO 9999 conceitua TA, também conhecida como Ajudas Técnicas, como "[...] qualquer produto, instrumento, estratégia, serviço e prática, utilizado por pessoas com deficiência e pessoas idosas, especialmente produzido ou geralmente disponível para prevenir, compensar, aliviar ou neutralizar uma deficiência, incapacidade ou desvantagem e melhorar a autonomia e a qualidade de vida dos indivíduos". (ISO 9999).

Segundo Bersch e Pelosi (2006, p. 08), "Ajudas Técnicas e Tecnologia Assistiva são expressões sinônimas quando se referem aos recursos desenvolvidos e disponibilizados às pessoas com deficiência e que visam ampliar suas habilidades no desempenho das funções pretendidas", sendo a Tecnologia Assistiva um conceito abrangente que busca promover a autonomia e independência funcional do seu usuário.

Ajudas Técnicas podem ser de grande complexidade, que envolvam tecnologias, ou podem ser simples, como por exemplo utensílios adaptados para alimentação. As tecnologias, de uma maneira geral, facilitam atividades do cotidiano. Para pessoas com deficiência, a TA proporciona "maior independência, qualidade de vida e inclusão através da ampliação de sua comunicação, mobilidade, controle de seu ambiente, habilidades do seu aprendizado e trabalho" (BERSCH, 2013, p.03).

\subsection{A Mobilidade por pessoas com Deficiência Visual}

Para o cego, a estrutura de navegação baseia-se no conhecimento da rota, tornando seu desafio maior em ambientes novos e desconhecidos. Para 
Melo (1991, p.37) existem algumas técnicas para que a orientação seja de forma segura. A primeira delas é que a pessoa com deficiência visual reconheça ou esteja familiarizado com o ambiente, não apenas em relação aos aspectos do ambiente como forma geométrica, quantidade de andares, corredores e salas, mas principalmente em relação a segurança: é importante que se saiba identificar prontamente as saídas de emergência e os locais de perigo. Além disso, é preciso que se saiba a localização de janelas, o tipo de piso, a localização dos banheiros, das escadas e quaisquer informações que possam ajudar na criação de uma imagem mental do ambiente. Esse conhecimento do ambiente ajuda a medir e comparar a distância dos ambientes e entre os objetos. A segunda técnica é o enquadramento ou alinhamento, que consiste em estabelecer uma posição no ambiente como ponto de partida e uma linha reta até o destino. Em ambientes desconhecidos, os pontos fixos de referência podem ser portas, paredes ou janelas; já em ambientes conhecidos, podem ser os objetos, desde que estes estejam fixos no local. Por fim, a terceira técnica é o rastreamento, que consiste em localizar objetos ou pontos de referência.

Pacientes com deficiências visuais graves enfrentam desafios assustadores na obtenção de mobilidade independente, e o deslocamento de um lugar para o outro é difícil e envolve um conjunto de tarefas. Uma técnica utilizada para deslocar-se de um lugar para o outro de forma independente e segura por pessoas com deficiência visual chama-se wayfinding, esta técnica consiste em duas perguntas básicas: onde estou? E para onde devo ir? Logo, saber a localização atual é tão importante quanto o destino. (SZETO, 2007, p.6360)

Hoje em dia, a solução da questão da mobilidade do cego está ligada à utilização de produtos de tecnologia assistiva, entre eles está a bengala longa, considerada como o mais efetivo e eficiente auxílio de locomoção (MELO, 1991, p.57). Ainda há a bengala laser, que funciona como um radar detectando obstáculos, no entanto, seu preço a torna inacessível.

No Brasil, a Norma Brasileira (NBR) 16537 regulamenta a questão da acessibilidade, incluindo diretrizes para instalação de pisos táteis. Esses pisos se caracterizam por relevos e luminâncias contrastantes em relação ao piso, formando um alerta ou uma linha-guia, servindo de orientação perceptível por pessoas com deficiência visual. Existe também a NBR 9050, que assegura a acessibilidade em edificações, no mobiliário, espaços e equipamentos urbanos.

Outra ajuda técnica conhecida é o cão-guia, que é um cão de assistência. Ele é adestrado para guiar a pessoa com deficiência visual em tarefas do cotidiano.

Além destas assistências conhecidas, existem alguns aplicativos e projetos que utilizam a tecnologia GPS para guiar pessoas com deficiência visual por locais externos, porém essa tecnologia não funciona em ambientes internos.

Para a navegação em ambientes internos, a Google lançou uma plataforma chamada Indoor Maps, que está ligada ao Google Maps e disponibiliza a planta de ambientes internos de aeroportos, estádios, shoppings e transporte público. Com as APIs (Application Programming Interface) disponibilizadas pelo Google, é possível que sistemas de navegação interna se apropriem destes recursos para a criação de um sistema de navegação eficiente para cegos. 


\subsection{Beacon, RFID E QR Code}

No processo inicial da pesquisa, com o objetivo de identificar os principais sistemas utilizados para a solução da problemática do artigo, utilizou-se de uma busca preliminar de forma exploratória para definir quais as tecnologias que seriam pesquisadas prioritariamente. Tendo em vista que as soluções apresentadas em artigos citam a utilização de Beacons, RFID e QR Code, decidiu-se que o recorte do artigo seria uma análise comparativa entre trabalhos que apresentassem essas tecnologias.

Os Beacons, apesar de recentes, vêm ganhando bastante destaque nos últimos tempos. Isto porque esse pequeno dispositivo, que significa "farol" em tradução literal, emite sinais por meio de tecnologia bluetooth low energy (BLE), que também é conhecida como bluetooth 4.0. Dessa maneira, boa parte dos smartphones e tablets usados atualmente podem captar os sinais que são interpretados como um gatilho para execução de alguma ação dentro de aplicativos. Segundo Freitas (2014), o bluetooth BLE apresenta dois grandes diferenciais. Um deles é a geração de ondas de rádio, o que permite maior qualidade de sinal em ambientes internos mesmo quando estes apresentam barreiras físicas como paredes, se diferenciando das redes WiFis que perdem potência de sinal conforme encontram essas barreiras. Além dessa vantagem, o bluetooth BLE apresenta baixo consumo de energia se comparado ao bluetooth tradicional. Adicionalmente, os Beacons funcionam em dispositivos iOS, Android, Windows ou qualquer dispositivo com bluetooth 4.0 ou superior. Segundo Teixeira (2014), essa conexão entre dispositivos pode-se dar de forma ativa ou passiva, sendo que a passiva, por exemplo, é simplesmente o armazenamento de que a conexão aconteceu, já a ativa é quando acontece uma interação com o dispositivo do usuário. De forma geral, o trabalho de inteligência dos Beacons é feito pelos aplicativos, ele não armazena conteúdos. Ainda para Teixeira (2014, p.1) o funcionamento dos Beacons de forma resumida, seria:

- O Beacon transmite uma localização para o aplicativo (ex: sensor código 190230124);

- O app no smartphone/smartwatch/device então se comunica com um servidor, que traduz aqui para ele (ex: 190230124 = corredor das batatas);

- O servidor verifica se há alguma ação a ser tomada quando essa proximidade acontece;

- Se houver alguma ação que deva ser tomada, o servidor manda para o aplicativo (ex: "batatas em promoção, enviar notificação para o usuário");

- O app, finalmente, exibe para o usuário uma mensagem amigável (ex: "aproveite o desconto de $30 \%$ nas batatas, só essa semana!").

Segundo o Freitas (2014), os Beacons funcionam enviando pequenos bits, com poucas informações, algo parecido com o endereço IP (Internet Protocol) ou uma coordenada GPS (Global Positioning System). Esses números consistem em uma UUID (Universal Unique Identifier) que contém as informações do fabricante do beacon e informa a região e a sub-região no qual está posicionado. Outra característica dos Beacons é que não possuem capa- 
cidade de armazenamento ou transmissão de grande quantidade de dados. Ou seja, os beacons são como um GPS indoor que possuem sua localização de forma precisa.

Hoje em dia, uma das principais utilizações dos Beacons está ligada à IoT (Internet of Things) na área do comércio com o intuito de venda. Uma das funções conhecidas dos Beacons, segundo Bottoni e Nishida (2014), é a capacidade de localizar com precisão por qual gôndola um cliente está próximo e enviar ofertas personalizadas com base em seu histórico de compras em relação àquela marca. De acordo com Ruffo (2014, p.1), entrevistado por Bottoni e Nishida (2014), além de serem extremamente precisos e possibilitarem a distinção entre dois sensores a centímetros de distância, "Agora é possível identificar o consumidor que entra na loja, conhecer o seu trajeto ao caminhar pelas seções e apresentar, baseadas em seus hábitos de consumo, ofertas e informações exclusivas para cada produto com uma agilidade impressionante". Além dessa funcionalidade, o STRAVA, um aplicativo de monitoramento de exercício físicos (corrida e ciclismo), oferece o uso de Beacons para segurança do atleta, podendo o usuário informar sua posição em tempo real para três pessoas. Essas são algumas das funcionalidades do Beacon, entre inúmeras que podem ser adicionadas. O próprio Ruffo (2014) diz "Até nossas casas poderão ser automatizadas com os Beacons. Imagine um sensor em sua geladeira, por exemplo, que ao se aproximar dispare o aplicativo do supermercado para que você monte a lista de compras, imagina. Ao chegar ao supermercado, o aplicativo pode direcioná-lo às gôndolas dos produtos contidos na lista, oferecendo as marcas selecionadas pelo varejista". Em relação a esta pesquisa, este é um dos maiores potenciais visíveis para a utilização dos Beacons por pessoas com deficiência visual.

O RFID (Radio-Frequency Identification), segundo o site do RFID-CoE (2016, p.1), "é uma tecnologia utilizada para identificar, rastrear e gerenciar desde produtos e documentos até animais ou mesmo indivíduos, sem contato e sem a necessidade de um campo visual. [...] A possibilidade de ser aplicada a inúmeras situações tornou a tecnologia RFID objeto de diversos projetos-piloto, em diferentes lugares no mundo". Ainda para o RFID-CoE (2016, p. 1), o RFID é uma tecnologia de identificação que utiliza a radiofrequência para capturar os dados, permitindo que uma tag seja lida sem a necessidade de campo visual, através de barreiras e objetos tais como madeira, plástico e papel. Um sistema RFID digital funciona como um sistema poderoso de aquisição de dados em tempo real, com a vantagem de eliminação de intervenções humanas manuais e visuais. É um método para armazenar e recuperar remotamente dados usando dispositivos denominados RFID tags ou transponders e existem dois tipos diferentes de tag, que pode ser passivo e ativo. No caso das tags passivas, elas não possuem fonte de alimentação incorporada se as informações são gravadas permanentemente, contudo há tags regraváveis. Já as a tags ativas possuem esta fonte de alimentação incorporada que fazem seu sinal ser transmitido por uma distância maior e seu armazenamento também ser maior (MOOI; ENG \& ZULKIFLI, 2010, p.1,). 
Para Retscher e Fu (2010), a tecnologia RFID tem as seguintes vantagens:

- Uma maior faixa de leitura/escrita em comparação com a maioria das outras tecnologias de identificação para equipamentos de longo alcance;

- RFID é barato, fácil e rápido de instalar para aplicações de curto tempo (como, por exemplo, em museus, salas de exposição, apresentações), porque não há necessidade de rede;

- Não precisa estar no campo visual;

- Pode ser usado para determinar posições e rastreamento;

- Pode ser usado para verificar a presença de pessoas e objetos.

Segundo o RFID-CoE (2016), a RFID tag é mais eficaz se for necessário um processamento rápido, de maior alcance e leitura, capacidade de transporte de dados flexível e transações mais seguras se for comparado à outras tecnologias. Além disso, o RFID é uma tecnologia que pode ser considerada acessível economicamente. Entre as aplicações do RFID tag, destacam-se segurança e controle de acessos, controle de tráfego de veículos, identificação pessoal, rastreamento animal e identificação de objetos. Suas áreas de aplicação são igualmente as mais variadas: setor público (controle de passaportes, identificação de ativos em bibliotecas), farmacêutico (autenticidade de produtos), automotivo (imobilizador eletrônico de motor), varejista (controle do fluxo de mercadoria), aéreo (identificação e movimentação de bagagens em aeroportos), médico-hospitalar (identificação de pacientes, controle da administração de medicamentos) entre outros (RFID-COE 2016, p. 1).

O QR Code é um código de barras em 2D, que pode ser escaneado por dispositivos com smartphones e tablets que possuam câmera, e nesse código contém a codificação de textos, ou URL (Uniform Resource Locator). A vantagem desse tipo de codificação é a capacidade de armazenamento de caracteres, que é bem maior que a do código de barras. Para caracteres numéricos ele armazena até 7.089, para alfanumérico 4.296 e para binário (8bits) 2.953 caracteres. Outra vantagem do $Q R$ code é o fato de ter uma redundância em sua codificação, o que torna possível uma leitura bem-sucedida mesmo quando são feitas imagens parciais do código. Detalhes sobre os produtos reconhecidos podem ser codificados nos próprios códigos ou recuperados a partir do back-end (LÓPEZDE-IPIÑA; LORIDO; LÓPEZ, 2011, p.38). Além disso, essa codificação possui a capacidade de correção de erros, ou seja, os dados podem ser restaurados mesmo se o símbolo estiver parcialmente sujo ou danificado.

\section{6 Áreas Comerciais}

Muitas alternativas estão sendo estudadas para permitir que a pessoa com deficiência visual faça compras no supermercado. Entre elas, os serviços de compra pela internet e entrega em domicílio são interessantes, no entanto, esses serviços em domicílio apresentam desvantagens relevantes: a independência pessoal é reduzida e não há possibilidade de compras não 
planejadas (NICHOLSON; KULYUKIN; COSTER, 2009).

Kulyukin e Kutiyanawala (2010), analisando sistemas de compras assistivas já desenvolvidos, identificaram alguns requisitos de design ideais para estabelecimentos facilitarem a compra por pessoas com deficiência visual. Primeiramente, é necessária a possibilidade de "seleção de produtos via smartphone", para que a pessoa com deficiência visual possa conhecer e navegar através dos produtos existentes no estabelecimento antes mesmo de iniciar a compra. Também é preciso que o deficiente possa "navegar pela loja", a fim de localizar a seção desejada facilmente. Outro requisito importante é a "busca e identificação do produto", onde a pessoa com deficiência, já na seção do produto desejado, deve ser capaz de localizar o produto escolhido ou selecionar um produto entre os disponíveis na área de interesse. A pessoa com deficiência também deve ser capaz de "utilizar os dispositivos existentes" - neste caso, por exemplo, um telefone celular e uma bengala (dispositivos já conhecidos por ele) podem ser aprimorados a fim de facilitar a mobilidade e a compra.

Por outro lado, embora mudanças sejam necessárias, Kulyukin e Kutiyanawala (2010) salientam que é importante que haja "ajustes ambientais mínimos" no estabelecimento - modificações maiores podem ser complexas e gerar custos altos, o que não é interessante para o proprietário. A respeito desses "ajustes ambientais mínimos", López-de-Ipiña, Lorido e López (2011) também defendem que o comportamento comercial convencional da loja não seja alterado. Isso está sendo defendido principalmente porque os estabelecimentos estão relutantes em modificar sua estrutura de forma complexa, e, portanto, qualquer solução viável deve deixar os produtos como estão e prioritariamente explorar a estrutura já existente (LÓPEZ-DE -IPIÑA; LORIDO; LÓPEZ, 2011).

\section{Materiais e Métodos}

O método utilizado para dar suporte ao desenvolvimento do presente estudo foi a revisão integrativa sistemática. Segundo Ferenhof e Fernandes (2016), esse tipo de revisão é uma investigação científica que visa eliminar vieses por meio do planejamento e sistematização de busca em bases de dados científicas por estudos originais, sintetizando os resultados em um portfólio bibliográfico. Foi, então, formulada a seguinte questão de pesquisa para este estudo: Quais tecnologias de navegação indoor e identificação de objetos existentes atualmente podem ajudar na localização e no conhecimento dos produtos dentro de um supermercado por uma pessoa com deficiência visual? Ou seja, quais tecnologias poderiam ajudar uma pessoa com deficiência visual a fazer compras sem o auxílio de uma pessoa vidente que também auxiliasse na navegação na área interna do supermercado?

A partir da definição do tema a ser pesquisado, organizou-se o processo da revisão integrativa sistemática a partir das etapas de coleta de dados, análise de dados e síntese. Na primeira parte do estudo, fez-se a identificação dos critérios de seleção e exclusão (figura 1), determinando quais se- 
riam as palavras chave e os termos da pesquisa. Para os critérios de seleção, era necessário que os artigos apresentassem alguns destes itens:

- Apresente pelo menos um destes hardwares: beacon, RFID ou QR Code;

- O auxílio ao usuário com deficiência visual apresente-se em mensagem de áudio ou mensagem de texto;

- Apresente auxílio na navegação e na identificação dos produtos;

Para os critérios de exclusão, era necessário que os artigos não apresentassem estes itens:

- Não fossem de vestir ou relacionados ao uso da bengala;

- Não fosse relacionado a criação das tecnologias;

- Não fossem artigos relacionados à saúde.

As palavras chaves deveriam permitir a busca do tema proposto, por isso, foram selecionadas as seguintes palavras: indoor, navigation, blind, visual impairment, beacon, RFID e "QR code". De forma que elas obedecessem a seguinte query: indoor AND navigati* AND (blind OR visual impair*) AND (beacon OR RFID OR "QR code"). A pesquisa foi realizada no dia 3 de novembro de 2016 e foi definido que os artigos a serem analisados seriam de 2010 até a data da pesquisa. A busca foi realizada em quatro periódicos relacionados à área do Design: Scopus, Web of Knowledge, Science Direct e IEEE Xplorer. Nessa busca, foram encontrados 41 artigos, destes 12 eram duplicados, ou seja, se encontravam em mais de uma base de dados. Para a seleção dos artigos que interessavam ao escopo da pesquisa, usou-se os parâmetros de inclusão e exclusão previamente definidos e a criação de três filtros que serviram para conhecer o conteúdo dos artigos a serem analisados. Estes filtros foram: (a) a leitura do título e o resumo, (b) a leitura da introdução e conclusão e (c) a leitura completa dos artigos. Dos 29 artigos pré-selecionados, 19 não coincidiam com os parâmetros de inclusão e foram descartados da pesquisa.

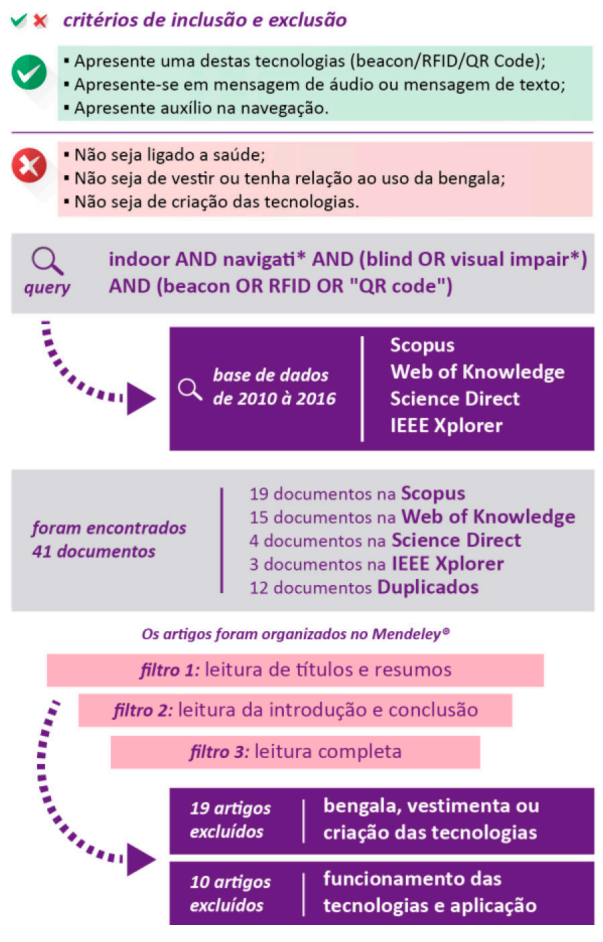

Figura 1: Sistematização do método utilizado. 
Assim, foram coletados os dados de 10 artigos (tabela 1) que estavam dentro do escopoda pesquisaquetemseu foconoentendimento do funcionamento dastecnologias, metodologias aplicadas, testes realizados e os resultados apresentados pelos autores.

Tabela 1: Artigos selecionados para a pesquisa.

\begin{tabular}{l} 
Artigo \\
\hline NavCog: turn-by-turn smartphone navigation \\
assistant for people with visual impairments or \\
blindness \\
NavCog: A Navigational Cognitive Assistant for \\
the Blind \\
Ray: Smart Indoor/Outdoor Routes for the Blind \\
Using Bluetooth 4.0 BLE
\end{tabular}

An efficient indoor navigation technique to find optimal route for blinds using $Q R$ codes

\begin{tabular}{lc}
\multicolumn{1}{c}{ Autores } & Ano \\
AHMETOVIC, D.; GLEASON, & 2016 \\
C.; KITANI, K. M. &
\end{tabular}

AHMETOVIC, D.; GLEASON， 2016 C.; KITANI, K. M.; TAKAGI, H.

CASTILLO-CARA, M.;

2016

HUARANGA-JUNCO, E.;

MONDRAGÓN-RUIZ, G.;

SALAZAR, A.; BARBOSA, L.

O.; ANTÚNEZ, E. A.

IDREES, A.; IQBAL, Z.;

ISHFAQ, M.

Accurate Positioning Using Long Range Active

RFID Technology to Assist Visually Impaired

ALGHAMDI, S.; SCHYNDEL, 2014

People

Indoor Navigational Aid Using Active RFID and

$Q R$-Code For sighted and Blind People

R. V.;KHALIL, I.

ALGHAMDI, S.; SCHYNDEL, 2013

R. V.; ALAHMADI, A.

RSNAVI: An RFID-based context-aware indoor

navigation system for the blind

Indoor Navigation and Product Recognition

for Blind People Assisted Shopping

IVANOV, $\mathrm{R}$.

2012

LOPEZ-DE-IPINA, D.;

LORIDO, T.; LOPEZ, U.

2011

INSIGHT: RFID and Bluetooth Enabled

GANZ, A.; GANDHI, S R;

2010

Automated Space for the Blind and Visually

Impaired

WILSON, C;

IMULLETT, G

Indoor navigation system for visually impaired

IVANOV, $\mathrm{R}$.

2010

Além dos artigos já mencionados que foram descobertos pela query nas bases de dados, foram encontrados também os artigos da tabela 2 que se relacionavam à pesquisa e entravam nos critérios de inclusão que não foram identificados na busca utilizando as palavras chave. Pretende-se como extensão desta pesquisa a inclusão da análise desses artigos, uma vez que tratam sobre o ato de fazer compras por pessoas com deficiência visual.

Após a seleção de todos os artigos que obedeceram ao escopo da pesquisa, os dados relacionados à tecnologia empregada, aos testes e procedimentos apresentados e o resultado obtidos pelo autor (figura 2) foram extraídos e organizado em forma de tabela para uma melhor estruturação e compreensão do sistema como um todo.

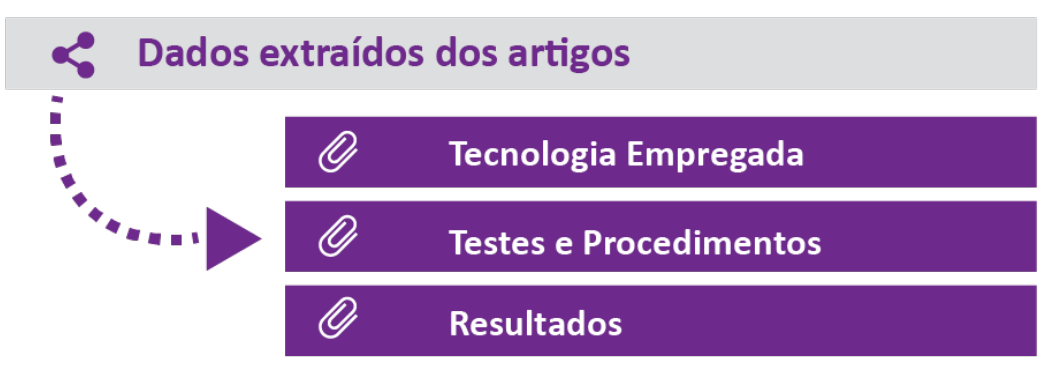

Figura 2: Sistemática de como foi realizado a tabulação dos dados apresentados nos artigos.

Tainá A. Bueno de Oliveira,

Flávio Anthero N. V. dos Santos, Milton José Cinelli

HFD, v.6, n.11, p 22-42, fev/jul 2017 


\section{Análise e Discussão dos Resultados}

Inicialmente, fez-se a comparação dos artigos de acordo com a tecnologia empregada. A tabela 2 mostra, de forma resumida, como cada sistema funciona e quais as tecnologias empregadas.

Tabela 2: Tecnologia e funcionamento dos sistemas empregados pelos artigos.

\begin{tabular}{ll}
\hline \multicolumn{1}{c}{ Artigo } & \multicolumn{1}{c}{ Tecnologia } \\
\hline (1) NavCog: turn-by-turn & O sistema apresenta uma estrutura com três \\
smartphone navigation assistant & componentes, sendo eles o NavCog App para iOS, o \\
for people with visual & servidor para os mapas e o Beacon BLE.
\end{tabular}
impairments or blindness

(2) NavCog: A Navigational O sistema apresenta uma estrutura com três Cognitive Assistant for the Blind componentes, sendo eles o NavCog App para iOS, o servidor para os mapas e o Beacon BLE.

(3) Ray: Smart Indoor/Outdoor Routes for the Blind Using Bluetooth 4.0 BLE

É um sistema híbrido para ambientes interno e externo com base no uso de tecnologias GPS e Bluetooth junto aos dispositivos móveis que se baseia em um micro controlador AVR e para comunicação, dispositivos Bluetooth 4.0 que podem operar em dois modos diferentes: Bluetooth Low Energy (4.0 BLE) e Enhanced Data Rate (EDR 2.0).

(4) An efficient indoor navigation technique to find optimal route for blinds using $Q R$ codes O sistema proposto requer um cenário personalizado com códigos QR anexados ao piso em forma de tiras horizontais e um smartphone Android com webcam integrada com versão OS 2.2 ou superior.

(5) Accurate Positioning Using Long Range Active RFID Technology to Assist Visually Impaired People O sistema consiste em um hardware que é composto por um leitor de RFID e um dispositivo móvel. E o software consiste no programa leitor e na API que contém o algoritmo da tag mais próxima e o algoritmo para área externa que estima a posição dos usuários.

(6) Indoor Navigational Aid Using Active RFID and QRCode For sighted and Blind People O sistema usado é quase um sistema omnidirecional. O sistema contém hardware e software. Os dispositivos de hardware trabalham de forma omnidirecional em uma escala de até 70 metros. 0 hardware é composto um leitor móvel de RFID, tags RFID ativas e $Q R$ Code. O software é composto API RFID ativa, $Q R$ Code reader e software de navegação.

(7) RSNAVI: An RFID-based O sistema possui tags RFID passivas e smartphones context-aware indoor navigation system for the blind que fazem a leitura de NFC. Ele é dividido em camada de construção que que é responsável pelos usuários, pelo rastreamento de objetos, identificação de coisas e monitoramento de status dos sensores. Camada de rede sendo sua função é entregar informações obtidas a partir da camada de 
(8) Indoor Navigation and Product Recognition for Blind People Assisted Shopping*

(9) INSIGHT: RFID and Bluetooth Enabled Automated Space for the Blind and Visually Impaired

(10) Indoor navigation system for visually impaired construção para serviços localizados na Internet e Camada de aplicação realiza a funcionalidade do sistema de navegação por um serviço WEB baseado em Java.

Suporte tecnológico para realização de compras de produto em supermercado por cegos.

BlindShopping oferece suporte para todo o processo de compra dentro um supermercado: (a) produto/navegação, (b) produto/seleção, (c) identificação do produto e (d) produto de busca. Funciona com um sistema de navegação com um leitor de RFID e tags RFID distribuída por corredores de supermercados que informa a navegação por meio de áudio no smartphone via bluetooth. Um sistema de reconhecimento do produto por captura do QR Code ou UPC (Universal Product Code) do produto. E gestão do sistema que é o gerenciamento via web das tags RFID e QR Code por uma interface RIA (Rich Internet Application) que pode ser acessada pelo navegador.

Um sistema de multi-resolução que pode rastrear o usuário utilizando tecnologia RFID passiva e Beacons (b-tracks) com um PDA e um leitor RFID. A arquitetura do sistema é composta por Ambiente: inclui etiquetas $R F I D$ passivas ( $R$-tags) e unidades de rastreamento bluetooth ( $B$-Tracks). Software Client: inclui o módulo de varredura $R$-tag, o módulo Bluetooth Zone Localization e o mecanismo Text2Speech para saída de áudio para o usuário.

Software Server: estrutura de banco de dados para $R$-tags e design do software Path Finding.

IndoorNavi é um sistema que trabalha com navegação a partir da posição do utilizador e identificação por voz. Há uma ativação automática da aplicação quando o celular se aproxima da etiqueta RFID. O sistema propõe-se a implementação da navegação de sala em sala. Para esta finalidade as etiquetas RFID são colocadas em cada porta. O sistema reconhece dois tipos de tags: as Navi Tags, que contêm informações de navegação e tags de áudio, que contêm mensagens de voz.

*Artigo que trata sobre navegação e compras por pessoas cegas

Para implementar uma rede de navegação interna, todos os artigos se basearam em uma ou mais tecnologias definidas como fator inclusivo da revisão bibliográfica integrativa. Algo interessante que se pode perceber é que tanto os Beacons quanto as RFID tags funcionam similarmente quando se trata de navegação. A diferença mínima que esses sistemas apresentam fica por conta da precisão na localização. Outro dado já apresentado 
no referencial bibliográfico e marcante aqui, é que esses hardwares, por assim dizer, funcionam integrados a um aplicativo que irá moldar tanto a estrutura de navegação, quanto, no caso do artigo (8), a estrutura para se fazer compras. A maioria dos artigos analisados trata apenas da questão de navegação indoor e apresentam como a utilização dessas tecnologias pode ser benéfica para usuários com deficiência visual.

Em relação a navegação, algo interessante de se ressaltar na comparação desses artigos é o fato de que boa parte deles utilizam RFID tags ativas, apesar de custarem mais em relação as RFID tags passivas por proporcionarem sinal de maior alcance. Entretanto, quando usada com Beacons, essas podem ser passivas, já que o sinal dos Beacons tem um alcance maior. Alguns artigos também abordam um leitor externo de RFID, contudo, atualmente o smartphone já possui aplicativos que fazem a leitura de RFID tags de forma que essa função seja praticamente incorporada não necessitando o gasto com mais um aparelho.

Há duas estratégias para a instalação de RFID. Todos os artigos apresentados utilizam a estratégia de baixo custo, que consiste na utilização de várias RFID tags e um leitor apenas. Nessa situação de navegação ela apresenta ser a mais apropriada. A estratégia de alto custo seria a utilização de vários leitores de RFID e uma tag, que normalmente é utilizada para controle e proteção de mercadorias em lojas.

Nos testes e procedimentos apresentado pelos autores, a intenção da maioria é que esses sistemas não apresentassem mudança estrutural nos ambientes internos, sendo assim, tanto Beacons quanto RFID tags foram colados ou em paredes ou no chão dos ambientes, nas distâncias calculadas para que esse pudesse guiar com o máximo de precisão o usuário cego. Foi notado por alguns autores que Beacons e RFID de marcas e fornecedores diferentes também variavam o campo de alcance.

Em relação à navegação, foi percebido que o arranjo dos Beacons e RFID estão posicionados é que determinará o sucesso da precisão no posicionamento. Segundo Alghamdi, Schyndel e Alahmadi (2013), existem três tipos de posicionamento: as Boundary Tags, que devem ser instalados nos cantos no interior do ambiente com o objetivo de desenhar o mapa para fins de navegação, incluindo evitar grandes obstáculos, determinar o caminho mais curto e, assim, ajudar os usuários a alcançar seus destinos; as Information Point Tag, que são tags de $Q R$ code que contêm informações gerais sobre uma determinada área da construção, como o nome do edifício, número do piso e nome do departamento; e as Points Of Interest, que são tags que identificam um local específico, como número de escritório, banheiro e saída de emergência.

Outro ponto importante ressaltado pela maioria dos autores é que nem sempre a rota mais curta é a melhor rota, e que um sistema bom é aquele que indica a melhor rota, com menos obstáculos ou perigos. Vale ressaltar que todos os sistemas apresentados se preocupam com essa ca- 
racterística. Alguns sistemas utilizam mapas dos ambientes, além de permitir que o usuário insira o seu próprio ambiente de forma que este esteja disponível na nuvem e possa ser feito seu download em um servidor localizado na internet. Algo que pode ser um potencial para esse sistema é o uso do Google Indoor. Apesar de ser uma ferramenta nova, com poucos recursos, com o advento dos Beacons é algo que pode ser potencialmente promissor, uma vez que a Google pode vir a fornecer os APIs para a utilização em aplicativos de desenvolvedores.

O sistema RSNAVI possui características bem interessantes que valem ser ressaltadas aqui. Esse sistema permite um modo de exploração onde o usuário cego recebe informações sobre: (1) apartamento ou escritório: nome, área, número de quartos, nomes dos quartos; (2) sala: nome, área, exposição, obstáculos e perigos. (3) elevador: alcance do chão, acessibilidade, posição dos botões; (4) porta: automática ou não, tipo deslizante ou giratório, estado (aberto, fechado, bloqueado, bloqueado); (5) descrição de todos os objetos (nomes e distâncias); (6) os nomes dos objetos circundantes mais próximos (frente, costas, esquerda, direita). Após o planejamento da rota, uma sequência de waypoints (ou point of interest) descrevem a rota até o ponto de destino. Além disso, os waypoints também descrevem um modo de navegação onde o usuário recebe comandos de como alcançar cada waypoint, por exemplo "siga a parede para do seu lado esquerdo até chegar a uma porta", "depois de cinco passos vire à esquerda". Esse sistema utiliza, assim, uma navegação baseada em pontos de referência em corredores longos e combinação de navegação baseada em indicadores e métricas nos quartos.

O BlindShopping, o IndoorNavi e o Ray, apresentam sistemas semelhante ao RSNAVI. De maneira geral, os autores apresentam de forma positiva a condução dos testes. As tecnologias atenderam as expectativas, sendo que as mais utilizadas foram os Beacons e RFID tags, provavelmente por sua ampla possibilidade de uso em diversas situações como salientam as pesquisas. Os $Q R$ Codes também são alternativas interessantes, porém, apresentam menos interação se consideradas as possibilidades que os outros expressam.

Uma vez que o presente artigo se propôs a elencar quais dessas tecnologias poderiam ser viáveis para que uma pessoa com deficiência visual pudesse fazer compras de forma independente, toma-se como base as diretrizes propostas por Kulyukin e Kutiyanawala (2010), que sugerem que as mudanças nos ambientes comerciais devem ser mínimas. Nesse contexto, todas a tecnologias abordadas nesse artigo --Beacons, RFID tags e $Q R$ Code -- são ideais, pois nenhuma precisa de mudanças drásticas no ambiente, além de serem dispositivos pequenos.

Em contrapartida, na possibilidade de "seleção de produtos via smartphone", os Beacons e os RFID tags oferecem mais recursos, quando comparados ao $Q R$ Code. Um dos recursos que poderia ser explorado de for- 
ma bastante eficaz (com o auxílio de um aplicativo programado para essa tarefa) é o envio de informação dos produtos posicionado nas gondolas por RFID tag ativo, de forma que o usuário pudesse determinar em seu aplicativo como seria expressa essa informação - do produto mais barato ao mais caro, ou por marca, por exemplo. Como a RFID tag possui um sinal que atinge menores distâncias que os Beacons, seria interessante que essa tecnologia fosse utilizada principalmente para a identificação dos produtos e enviando sinais de produtos que estão nas prateleiras. Em conjunto com essa tecnologia, os Beacons poderiam servir para definir o trajeto dentro do supermercado, ou para a navegação pela loja, a fim de localizar a seção desejada facilmente. O uso dos Beacons poderia auxiliar na utilização das RFID tag, uma vez que seu sinal tem maior alcance que esta última (seriam necessários por exemplo, menos Beacons que RFID tags para demarcar um trajeto). Por ser uma tecnologia considerada bastante precisa, com a ajuda do aplicativo, poderiam ser traçadas rotas não só curtas, mas também seguras.

Um aplicativo seria o suporte necessário para que esses dois dispositivos funcionassem bem. Com o uso de um aplicativo poderiam ser inseridas diversas funcionalidades, inclusive que fossem personalizáveis a gosto do usuário. Outro potencial para as RFID tags e Beacons em conjunto com um aplicativo seria que, antes mesmo de entrar no supermercado, o usuário já pudesse selecionar os produtos que necessita comprar e o aplicativo definir a rota necessária para executar essa tarefa.

O que torna esse sistema viável para o usuário é o fato de poder usar o próprio smartphone sem a necessidade de outros dispositivos. Além disso, para o comerciante esse sistema pode ser implantado por valores acessíveis perto de outras soluções. Esse sistema não só beneficiaria pessoas com deficiência visual, mas também idosos na localização de produtos e até o resto da população como forma de ganhar tempo com uma rota definida e com informação das promoções.

É evidente que essas sugestões são uma pequena parte do que se pode fazer utilizando RFID tags e Beacons, entretanto, já é o começo para a discussão da promoção de autonomia de pessoas cegas.

\section{Considerações Finais}

Esta pesquisa se propôs, como objetivo geral, analisar de forma sistematizada bibliografias que demonstram as contribuições das tecnologias de navegação indoor e identificação de objetos, e que estas pudessem ser utilizadas no contexto do supermercado a fim de permitir que pessoas com deficiência visual pudesse, de maneira autônoma, circular e fazer compras de forma segura. Pode-se chegar assim à algumas considerações: existe público para esse tipo de tecnologia, tanto a quantidade de pessoas que possuem algum tipo de limitação visual quanto para idosos; são tecnologias viáveis e que possuem um custo de imple- 
mentação acessível, não necessitando por parte do usuário um gasto a mais e por parte do comerciante pequenas alterações no estabelecimento. Outro benefício é que a implementação desse recurso por parte do comerciante pode ser usada também com os demais usuários que não necessitam de suporte, na divulgação de promoções entre outras inúmeras possibilidades.

O presente artigo também abre a discussão acerca da ponte entre o físico e o digital de uma forma diferenciada do que se conhece. Atualmente, uma boa aplicação são as lojas online, que mantém uma boa base de dados em relação ao consumo de seus usuários. Essa experiência pode também fazer parte do mundo físico, de forma que facilite o acesso por pessoas que tenham algum tipo de dificuldade, oferecendo às mesmas facilidades e experiências da internet com a vantagem de poder experimentar o produto.

Essas são algumas das considerações que se pode ponderar com o uso desse tipo de tecnologias. Em relação às pesquisas futuras, sugere-se que se acrescente na análise os artigos presentes na tabela 3 que se relacionam à pesquisa e contemplam os critérios de inclusão, mas que não foram encontrados na busca utilizando as palavras chave escolhidas. Além dessa inclusão na análise, sugere-se também o teste utilizando as tecnologias sugeridas, inicialmente em ambiente simulado com a presença de usuários para que seja validado as considerações comparativas presentes nesse artigo.

Tabela 3: Artigos acrescentados à pesquisa.

\begin{tabular}{|c|c|c|}
\hline Artigo & Autores & Ano \\
\hline $\begin{array}{l}\text { Mobile Vision as Assistive Technology for the } \\
\text { Blind: An Experimental Study }\end{array}$ & MANDUCHI, R. & 2012 \\
\hline $\begin{array}{l}\text { Indoor Navigation for the Blind and Vision } \\
\text { Impaired: Where are we and Where are we } \\
\text { Going? }\end{array}$ & $\begin{array}{l}\text { WISE, E.; LI, B.; } \\
\text { GALLAGHER, T.; } \\
\text { DEMPSTER, A. G.; RIZOS, } \\
\text { C.; RAMSEY-STEWART, E.; } \\
\text { WOO, D. }\end{array}$ & 2012 \\
\hline $\begin{array}{l}\text { BlindShopping: Enabling Accessible Shopping for } \\
\text { Visually Impaired People through Mobile } \\
\text { Technologies }\end{array}$ & $\begin{array}{l}\text { LOPEZ-DE-IPINA, D.; } \\
\text { LORIDO, T.; LOPEZ, U. }\end{array}$ & 2011 \\
\hline $\begin{array}{l}\text { Accessible Shopping Systems for Blind and } \\
\text { Visually Impaired Individuals: Design } \\
\text { Requirements and the State of the Art }\end{array}$ & $\begin{array}{l}\text { KULYUKIN, V; } \\
\text { KUTIYANAWALA, A. }\end{array}$ & 2010 \\
\hline $\begin{array}{l}\text { ShopTalk: Independent Blind Shopping Through } \\
\text { Verbal Route Directions and Barcode Scans }\end{array}$ & $\begin{array}{l}\text { NICHOLSON, J.; } \\
\text { KULYUKIN, V.; COSTER, D. }\end{array}$ & 2009 \\
\hline $\begin{array}{l}\text { ShopTalk: Independent Blind Shopping =Verbal } \\
\text { Route Directions + Barcode Scans }\end{array}$ & $\begin{array}{l}\text { NICHOLSON, J.; } \\
\text { KULYUKIN, V. }\end{array}$ & 2007 \\
\hline $\begin{array}{l}\text { Trinetra: Assistive Technologies for Grocery } \\
\text { Shopping for the Blind }\end{array}$ & $\begin{array}{l}\text { LANIGAN, P. E.; PAULOS, } \\
\text { A. M.; WILLIANS, A. W.; } \\
\text { ROSSI, D.; NARASIMHAN, } \\
\text { P. }\end{array}$ & 2006 \\
\hline $\begin{array}{l}\text { RoboCart: Toward Robot-Assisted Navigation of } \\
\text { Grocery }\end{array}$ & $\begin{array}{l}\text { KULYUKIN, V.; GHARPURE, } \\
\text { C.; NICHOLSON, J. }\end{array}$ & 2005 \\
\hline
\end{tabular}




\section{REFERÊNCIAS}

BERSCH, R. de C. R; PELOSI, M. B. Portal de ajudas técnicas para educação: equipamento e material pedagógico para educação, capacitação e recreação da pessoa com deficiência física: tecnologia assistiva: recursos de acessibilidade ao computador II / Secretaria de Educação Especial - Brasília: ABPEE - MEC : SEESP, 2006. Disponível em: <http://www.fipa.com.br/facfipa/ pdf/MEC_Tecnologia_Assistiva.pdf>. Acesso em: 3 Jan. 2016.

BERSCH, R. de C. R. Introdução à Tecnologia Assistiva. Porto Alegre. 2013. Disponivel em: <http://www.assistiva.com.br/Introducao_Tecnologia_Assistiva.pdf>. Acesso em: 4 Fev. 2016.

BOTTONI, F.; NISHIDA, E.. PROXXIMA/Meio Mensagem. Beacons: pega ou não pega? Tecnologia de localização indoor adotada pelas lojas Apple têm grandes entusiastas, mas ainda não é unanime. Disponível em: < http://www. proxxima.com.br/home/proxxima/noticias/2014/06/02/beacons-pega-ou -n-o-pega.html>. Acesso em: 26 Nov. 2016.

BRASIL. Decreto no 5.296 de 2 de dezembro de 2004. Disponível em: <http://www.planalto.gov.br/ccivil_03/_ato2004-2006/2004/decreto/ d5296.htm>. Acesso em: 20 Jan. 2016.

CENSO DEMOGRÁFICO 2010. IBGE - Instituto Brasileiro de Geografia e Estatística. Características gerais da população, religião e pessoas com deficiência. Disponível em: <http://biblioteca.ibge.gov.br/visualizacao/periodicos/94/cd_2010_religiao_deficiencia.pdf>. Acesso em: 20 Jan. 2016.

CIF. Classificação Internacional de Funcionalidade, Incapacidade e Saúde. Disponível em: <http://www.inr.pt/uploads/docs/cif/CIF_port_\%202004. pdf>. Acesso em: 30 Jan. 2016.

ENDEAVOR BRASIL. Beacon: o GPS que ajuda sua marca a localizar as melhores oportunidades. Disponível em: <https://endeavor.org.br/beacon/? esvt =-b\&esvq=_cat\%3Aendeavor.org.br\&esvad $\mathrm{t}=999999-=-1-$ \&esvcrea75514462525=\&esvplace=\&esvd=c\&esvaid50078=\&gclidCjwKEAiAyO_BBRDOgM-K8MGWpmYSJACePQ9CFk3Zy-ajGBO-G_2os0IdXOvTpaC4VQLPjD4r_PFiGxoCKbLw_wcB>. Acesso em: 26 Nov. 2016.

FREITAS, T. de. XDS. Beacons - 5 coisas que você precisa saber. Disponível em: <http://www.xds.com.br/beacons-5-coisas-que-voce-precisa-saber/>. Acesso em: 26 Nov. 2016.

ISO 9999, NORMA INTERNACIONAL. Disponível em: <http://www.siva. it/ftp/en_iso_9999.zip>. Acesso em: 22 Jan. 2016. (Tradução do autor)

KULYUKIN, V.; KUTIYANAWALA, A. Accessible Shopping Systems for Blind and Visually Impaired Individuals: Design Requirements and the State of the Art. The Open Rehabilitation Journal. v. 3, p.158-168, 2010. Disponível em: <http://www.novascholar.com/pdfs/citeseerx/citeseerx23/ours/570638. pdf>. Acesso em: 26 Nov. 2016.

LÓPEZ-DE-IPIÑA, D.; LORIDO, T.; LÓPEZ, U.. BlindShopping: Enabling Accessible Shopping for Visually Impaired People through Mobile Technologies. Proceedings of the 9th International Conference on Smart Homes and 
Health Telematics, Montreal, Canada, LNCS 6719. p. 266-270, 2011. Disponível em: <https://www.researchgate.net/publication/221338742_BlindShopping_Enabling_Accessible_Shopping_for_Visually_Impaired_People_ through_Mobile_Technologies>. Acesso em: 26 Nov. 2016.

LÓPEZ-DE-IPIÑA, D.; LORIDO, T.; LÓPEZ, U.. Indoor Navigation and Product Recognition for Blind People Assisted Shopping. Ambient Assisted Living, vol. 6693, pp. 33-40, 2011. Disponível em: <http://link-springer-com. ez74.periodicos.capes.gov.br/chapter/10.1007/978-3-642-21303-8_5\#page-1>. Acesso em: 26 Nov. 2016.

MELO, H. F. R. Deficiência Visual: Lições Práticas de Orientação e Mobilidade. Ed. UNICAMP: Campinas, 1991.

MICHAELIS. Disponível em: <http:// http://michaelis.uol.com.br>. Acesso em 27 de janeiro de 2016.

MOOI, W. S.; ENG, T. C.; ZULKIFLI, N. H. bt. N.. Efficient RFID Tag Placement Framework for In Building Navigation System for the Blind. Disponivel em: <http://ieeexplore.ieee.org.ez74.periodicos.capes.gov.br/stamp/stamp. jsp?arnumber $=5532064>$. Acesso em: 23 Nov. 2016.

NBR ABNT - ASSOCIAÇÃO BRASILEIRA DE NORMAS TÉCNICAS. Norma Brasileira 16537. Disponível em: <http://www.pessoacomdeficiencia.gov.br/ app/sites/default/files/arquivos/\%5Bfield_generico_imagens-filefield-description\%5D_168.pdf>. Acesso em: 26 Nov. 2016.

NBR ABNT - ASSOCIAÇÃO BRASILEIRA DE NORMAS TÉCNICAS. Norma Brasileira 9050. Disponível em: <http://www.pessoacomdeficiencia.gov.br/ app/sites/default/files/arquivos/\%5Bfield_generico_imagens-filefield-description\%5D_24.pdf>. Acesso em: 26 Nov. 2016.

NICHOLSON, J.; KULYUKIN, V.; COSTER, D. On Sufficiency of Verbal Instructions for Independent Blind Shopping. Proceedings of the 24th Annual International Technology and Persons with Disabilities Conference (CSUN). Los Angeles, CA, 2009. Disponível em: <https://www.researchgate.net/publication/255575663_ON_SUFFICIENCY_OF_VERBAL_INSTRUCTIONS_FOR_ INDEPENDENT_BLIND_SHOPPING>. Acesso em: 26 Nov. 2016.

PORTAL BRASIL. OMS diz que mais de 1 bilhão de pessoas no mundo sofrem de algum tipo de deficiência. Disponível em: <http://www.brasil.gov.br/ ciencia-e-tecnologia/2011/06/oms-diz-que-mais-de-1-bilhao-de-pessoas-no-mundo-sofrem-de-algum-tipo-de-deficiencias. Acesso em: 15 Nov. 2016.

QRCODE.COM. What is QR Code. Disponivel em: <http://www.qrcode. com/en/about/>. Acesso em: 26 Nov. 2016.

RETSCHER, G.; FU, Q.. Continuous Indoor Navigation with RFID and INS. Disponível em: <http://ieeexplore.ieee.org.ez74.periodicos.capes.gov.br/ stamp/stamp.jsp?arnumber $=5507242>$. Acesso em: 23 Nov. 2016.

RFID-CoE. O que é RFID. Disponível em: < http://www.rfid-coe.com. br/_Portugues/OqueERFID.aspx>. Acesso em: 23 Nov. 2016.

SZETO, A. Y. J.; SHARMA, S. K.. RFID Based Indoor Navigational Aid for 
Persons with Severe Visual Impairments. Proceedings of the 29th Annual International Conference of the IEEE EMBS, p.6360-6363, 2007. Tradução do autor.

TEIXEIRA, F. UXDESIGN.CC BRASIL. Tudo o que você precisa saber para começar a brincar com iBeacons. Disponível em: < https://brasil.uxdesign.cc/ tudo-o-que-voc\%C3\%AA-precisa-saber-para-come\%C3\%A7ar-a-brincarcom-ibeacons-fdf5847e640b\#.exk0gqzf5>. Acesso em: 26 Nov. 2016.

\section{AGRADECIMENTOS}

Este trabalho foi realizado durante o mestrado em Design pela Universidade do Estado de Santa Catarina/UDESC financiado com uma bolsa de estudos fornecida pela CAPES - Coordenação de Aperfeiçoamento de Pessoal de Nível Superior vinculada ao Ministério da Educação (MEC) do Brasil. 